\section{Diabetes mellitus Typ 2}

H. Hauner, W. A. Scherbaum

Deutsches Diabetes- Forschungsinstitut an der Heinrich-Heine-Universität Düsseldorf

\section{Type 2 diabetes}

Type 2 diabetes represents the most common form of carbohydrate disorders affecting at least $5 \%$ of the population in the industrialized world. In addition to genetic predisposition obesity and a sedentary lifestyle are the main promoters. Recent data clearly suggest that lifestyle intervention in people at increased risk can potently reduce the incidence of the disease. In overt type 2 diabetes, good glycaemic control and aggressive management of associated cardiovascular risk factors may prevent its multiple and costly complications.

\section{Epidemiologie}

Der Diabetes mellitus Typ 2 ist die bei weitem häufigste Diabetesform. Ca. $90 \%$ aller Menschen mit Diabetes mellitus sind diesem Typ zuzuordnen. Nach Hochrechnungen leiden derzeit etwa 5\% aller Bundesbürger an einem bekannten Typ2-Diabetes (ohne Dunkelziffer!) (Dtsch Med Wochenschr 1998; 123: 777-782). Die Krankheit beginnt üblicherweise im mittleren oder höheren Lebensalter. In den letzten Jahren wurde beobachtet, dass immer mehr junge Erwachsene und sogar Jugendliche betroffen sind (Diabetes Care 2000; 23: 381-389).

\section{Pathophysiologie}

Als Hauptursache für den weltweiten Trend einer Zunahme des Typ-2-Diabetes mellitus wird die Adipositas angesehen. Es besteht ein sehr enger Zusammenhang zwischen Körpergewicht und Diabetesrisiko. Das Diabetesrisiko nimmt mit zunehmender Körperfettmasse, Dauer der Adipositas sowie abdominellem Fettverteilungsmuster zu. Eine Gewichtszunahme erhöht das Diabetesrisiko, eine Gewichtsabnahme senkt sie (Ann Intern Med 1995; 122: 481-486). Vor allem eine Vermehrung der viszeralen Fettdepots ist eng mit Typ-2-Diabetes mellitus und anderen metabolischen Störungen assoziiert (Brit Med J 2001; 322: 716-720). Nach Schätzungen könnten zwei Drittel aller Diabetesfälle vermieden werden, wenn es gelänge, einen Anstieg des Körperge- wichts im Erwachsenenalter zu vermeiden.

Auch die Zusammensetzung der Ernährung hat einen direkten Einfluss auf das Diabetesrisiko. Vor allem eine kalorienreiche Kost mit einem hohen Anteil an gesättigten Fettsäuren und einer niedrigen Zufuhr von Ballaststoffen, die für Mitteleuropa und Nordamerika typisch ist, scheint das Diabetesrisiko zu erhöhen. Dagegen schützt eine Kost mit einfach und mehrfach ungesättigten Fettsäuren, reichlich Ballaststoffen und Kohlenhydraten mit niedrigem glykämischen Index vor Diabetes (J Amer Med Ass 1997; 277 : 472-477).

Daneben ist auch körperliche Bewegung für die Verminderung des Diabetesrisikos von Bedeutung. Körperlich aktive Menschen haben ein etwa halb so hohes Diabetesrisiko wie körperlich inaktive Menschen.

Falsche Ernährung und Bewegungsmangel können aber nur dann wirksam wer- den, wenn eine entsprechende genetische Prädisposition besteht. Nur für 510\% aller Personen mit Typ-2-Diabetes wurden bislang umschriebene Gendefekte identifiziert. Da die Diabetesgene noch zum größten Teil unbekannt sind, ist eine molekulargenetische Analyse derzeit wenig hilfreich. Dafür kann eine einfache Familienanamnese umso wertvollere Hinweise liefern. Bei einem Elternteil mit Typ-2-Diabetes müssen $30-40 \%$ der Nachkommen mit der Manifestation der Erkrankung im Laufe ihres Lebens rechnen.

Obgleich die Pathomechanismen, die zum Typ-2-Diabetes führen, längst nicht aufgeklärt sind, zeigt sich, dass Übergewicht, falsche Ernährung (hoher Anteil gesättigter Fettsäuren, ballaststoffarm) und Bewegungsmangel die Entwicklung einer Insulinresistenz fördern bzw. verstärken. Diese kann zunächst durch eine reaktiv gesteigerte Insulinsekretion des Pankreas kompensiert werden, erkennbar an erhöhten Seruminsulinkonzentrationen. Wenn allerdings die hohe Funktionsleistung vermutlich infolge genetischer Störungen nicht mehr aufrechterhalten werden kann und sich erschöpft, kommt es zur Manifestation der chronischen Hyperglykämie (N Engl J

\section{Info 1}

Ein erhöhtes Risiko für Typ 2-Diabetes besteht bei folgenden Parametern (Diabetes Care 1997; 20: 1183-1198):

- erstgradig Verwandte mit Typ 2-Diabetes mellitus

- Übergewicht (BMI $\geq 27 \mathrm{~kg} / \mathrm{m}^{2}$ )

- Hypertonie (RR $\geq 140 / 90 \mathrm{mmHg}$ )

- Dyslipoproteinämie (HDL-Cholesterin $\leq 35 \mathrm{mg} / \mathrm{dl}$, Triglyzeride $\geq 200 \mathrm{mg} / \mathrm{dl}$ )

- erhöhte Blutglukosewerte bei früheren Untersuchungen

- Schwangerschaftsdiabetes oder Geburt eines makrosomen Kindes 
tesmanifestationsrate bei Personen mit gestörter Glukosetoleranz zu senken. Die aktuelle Datenlage reicht aber nicht aus, um den Einsatz von Medikamenten zur Primärprävention des Typ-2-Diabetes empfehlen zu können.

\section{Sekundärprävention}

Menschen mit manifestem Typ-2-Diabetes haben ein hohes Risiko für mikround makroangiopathische Komplikationen, die nicht nur die Lebensqualität, sondern auch die Lebenserwartung der Betroffenen empfindlich beeinträchtigen können. Besonders hervorzuheben ist das erhöhte kardiovaskuläre Risiko von Typ-2-Diabetikern, das denen von Nichtdiabetikern nach vorangegangenem Myokardinfarkt entspricht (N Engl J Med 1998; 339: 229-234).

Bei Personen mit Typ-2-Diabetes kommt es daher darauf an, die Blutglukosewerte möglichst normnah einzustellen, um Mikroangio- und Neuropathie zu verhindern. Daneben müssen aber auch assoziierte Risikofaktoren wie Hypertonie oder Dyslipoproteinämie möglichst konsequent behandelt werden, um makroangiopathischen Komplikationen vorzubeugen.

Die United Kingdom Prospective Diabetes Studie (UKPDS) ergab, dass durch eine intensivierte blutglukosesenkende Therapie eine signifikante Reduktion der diabetes-assoziierten Komplikationen möglich ist (Lancet 1998; 352: 837853). Eine Senkung des HbA1c-Werts um $1 \%$ war mit einer Reduktion mikrovaskulärer Endpunkte um 37\%, tödlicher und nicht-tödlicher Herzinfarkte um $14 \%$ und tödlicher und nicht-tödlisonders gefährdet sind Personen mit einer genetischen Belastung (Eltern oder Geschwister mit Typ-2-Diabetes), Übergewicht (BMI $\geq 27 \mathrm{~kg} / \mathrm{m}^{2}$ ) oder Personen mit gestörter Glukosetoleranz (Info 1). Adipöse Personen mit familiärer Diabetesbelastung entwickeln mit 60- bis 70\%iger Wahrscheinlichkeit im Laufe ihres Lebens einen Diabetes.

Die wichtigsten Präventionsmaßnahmen sind Gewichtsreduktion bei Überrung haben sich in jüngster Zeit auch Medikamente wie Metformin, Acarbose, ACE-Hemmer, Statine und Orlistat als begrenzt wirksam erwiesen, um die Diabe-

\section{Info 2}

Sinnvolle Empfehlungen zur Prävention des Typ-2-Diabetes bei Personen mit gestörter Glukosetoleranz bzw. erhöhtem Diabetesrisiko (nach N Engl J Med 2001; 344: 1343-1350):

- Gewichtsreduktion > $5 \mathrm{~kg}$

- Reduzierung der Fettaufnahme $<30$ Energie \%

- Reduzierung der gesättigten Fette $<10$ Energie \%

- Steigerung der Ballaststoffaufnahme $>15 \mathrm{~g} / 1000 \mathrm{kcal}$

- körperliche Bewegung > 4 Stunden/Woche 
cher Schlaganfälle um $12 \%$ verbunden (Brit Med J 2000; 321: 405-412).

In der Hypertoniestudie der UKPDS ließ sich durch eine effektive Blutdrucksenkung mit dem ACE-Hemmer Captopril oder dem Betablocker Atenolol ebenfalls eine signifikante Senkung aller diabetesbezogenen Endpunkte um 24\%, der diabetesbezogenen Todesfälle um $32 \%$, der zerebralen Insulte um $44 \%$ und der mikrovaskulären Komplikationen um 37\% erzielen (Brit Med J 1998; 317: 713-720).

Durch diese und andere Studien gilt heute als gesichert, dass eine möglichst normnahe Diabeteseinstellung und strikte Behandlung begleitender Risikofaktoren von erheblichem Nutzen für die Betroffenen ist. Dies kommt auch in den neuesten Empfehlungen für die Behandlungsziele bzw. Einstellungskriterien von Menschen mit Diabetes mellitus Typ 2 zum Ausdruck (Info 3). Auch der Nutzen von niedrig dosierter Acetylsalicylsäure zur Prävention makroangiopathischer Komplikationen ist für Menschen mit Typ 2-Diabetes belegt.

\section{Ausblick}

Betrachtet man den Stellenwert der Präventivmedizin im deutschen Gesundheitssystem, ist zweifellos festzuhalten, dass die bereits heute verfügbaren Ansätze zur Prävention des Typ-2-Diabetes und seiner Komplikationen noch unzureichend genutzt werden. Dies dürfte zum Teil auf strukturelle Defizite, zum Teil aber auch auf ungenügende finanzielle Resourcen in der Primärprävention zurückzuführen sein.

Vor dem Hintergrund der heutigen Finanzierungsprobleme im Gesundheitswesen ist diese Situation unverständlich und untragbar, da der Typ-2-Diabetes zu den teuersten chronischen Erkrankungen überhaupt zählt. Besonders kostspielig sind dabei die vielfältigen Komplikationen eines unzureichend therapierten Diabetes. So verursachen Typ-2-Diabetiker mit mikro- oder makroangiopathischen Komplikationen etwa 2,5-fach höhere Kosten als GKV-Versicherte ohne Diabetes. Bei Typ-2-Diabetikern mit mikround makroangiopathischen Komplikationen liegen die Ausgaben sogar 4-fach höher (Dtsch Med Wochenschr 2001; 126: 585-589).

Bereits eine Verzögerung der Diabetesmanifestation bzw. der Komplikationen um wenige Jahre würde vermutlich erhebliche Kosten einsparen, die die Ausgaben für wirksame Präventions- und Be-

\section{Info 3}

Einstellungskriterien bei Diabetes mellitus Typ 2 mit niedrigem Risiko für mikro- und makrovaskuläre Komplikationen (nach Diab Stoffw 2002; im Druck):

- Blutglukose nüchtern 90-120 mg/dl; postprandial 130-160 mg/dl

$-\mathrm{HbA}_{1 \mathrm{c}} \leq 6,5 \%$

- Gesamtcholesterin $<200 \mathrm{mg} / \mathrm{dl}(<170 \mathrm{mg} / \mathrm{dl})^{*}$

- LDL-Cholesterin $<100 \mathrm{mg} / \mathrm{dl}(<100 \mathrm{mg} / \mathrm{dl})^{*}$

- HDL-Cholesterin $>35 \mathrm{mg} / \mathrm{dl}(>40 \mathrm{mg} / \mathrm{dl})^{*}$

- Triglyzeride $<150 \mathrm{mg} / \mathrm{dl}(<150 \mathrm{mg} / \mathrm{dl})^{*}$

- Blutdruck < 140/85 mmHg; mit Nephropathie $<130 / 80$ mm Hg

- Body Mass Index $<25 \mathrm{~kg} / \mathrm{m}^{2}$

* in Klammern: Diabetes mellitus Typ 2 mit Komplikationen handlungsprogramme bei weitem übersteigen dürften, zumal sich damit auch andere begleitende Risikofaktoren bessern lassen.

Die derzeit von der Deutschen Diabetes-Gesellschaft entwickelten evidenzbasierten Leitlinien sollen helfen, Prävention, Früherkennung und Behandlung des Typ-2-Diabetes auf der Grundlage des heutigen Kenntnisstandes zu optimieren (www.deutsche-diabetesgesellschaft.de).

Korrespondenz: Prof. Dr. H. Hauner

Klinische Abteilung, Deutsches DiabetesForschungsinstitut an der HeinrichHeine-Universität Düsseldorf Auf'm Hennekamp 65 40225 Düsseldorf

E-Mail: hauner@ddfi.uni-duesseldorf.de

\section{Fachliche Betreuung der „Serie Prävention“:}

Prof. Dr. Dr. h. c. Peter C. Scriba Medizinische Klinik / Klinikum Innenstadt der Universität München Ziemssenstr. 1

80336 München

Prof. Dr. Friedrich W. Schwartz Medizinische Hochschule Hannover Abteilung für Epidemiologie, Sozialmedizin und Gesundheitsforschung

Carl-Neuberg-Str. 1

30623 Hannover 